\title{
Nonlocal boundary value hyperbolic problems involving integral conditions
}

\author{
Allaberen Ashyralyev ${ }^{1,2}$ and Necmettin Aggez ${ }^{*}$
}

\author{
"Correspondence: \\ naggez@fatih.edu.tr \\ 'Department of Mathematics, Fatih \\ University, Istanbul, 34500, Turkey \\ Full list of author information is \\ available at the end of the article
}

\begin{abstract}
Stability estimates for the solution of the nonlocal boundary value problem with two integral conditions for hyperbolic equations in a Hilbert space $H$ are established. In applications, stability estimates for the solution of the nonlocal boundary value problems for hyperbolic equations are obtained.
\end{abstract}

MSC: $35 \mathrm{~L} 10$

Keywords: hyperbolic equation; stability; nonlocal boundary value problems

\section{Introduction}

It is well known that nonlocal boundary value problems with integral conditions are widely used for thermo-elasticity, chemical engineering, heat conduction, and plasma physics [1-4]. Some problems arising in dynamics of ground waters are defined as hyperbolic equations with nonlocal conditions [5] and [6]. The authors of [7] investigate nonclassical problems for multidimensional hyperbolic equation with integral boundary conditions and the uniqueness of classical solution. In [8] a linear second-order hyperbolic equation with forcing and integral constraints on the solution is converted to a nonlocal hyperbolic problem. Using the Riesz representation theorem and the Schauder fixed point theorem, existence and uniqueness of a generalized solution are proved. The solutions of hyperbolic equations with nonlocal integral conditions were investigated in [9-16]. The method of operators as a tool for investigation of the solution to hyperbolic equations in Hilbert and Banach spaces has been used extensively in [17-29].

In [30] the nonlocal boundary value problem

$$
\left\{\begin{array}{l}
\frac{d^{2} u(t)}{d t^{2}}+A u(t)=f(t), \quad 0 \leq t \leq 1, \\
u(0)=\sum_{r=1}^{n} \alpha_{r} u\left(\lambda_{r}\right)+\varphi, \quad u_{t}(0)=\sum_{r=1}^{n} \beta_{r} u_{t}\left(\lambda_{r}\right)+\psi \\
0<\lambda_{1} \leq \lambda_{2} \leq \cdots \leq \lambda_{n} \leq 1
\end{array}\right.
$$

was investigated. Stability estimates for the solution of the problem were established. First order of accuracy difference schemes for the approximate solutions of the problem were presented. Stability estimates for the solution of these difference schemes were established. Theoretical statements were supported by numerical examples.

C) 2014 Ashyralyev and Aggez; licensee Springer. This is an Open Access article distributed under the terms of the Creative Commons Attribution License (http://creativecommons.org/licenses/by/4.0), which permits unrestricted use, distribution, and reproduction in any medium, provided the original work is properly credited. 
In the present paper, we consider the nonlocal boundary value problem with integral conditions

$$
\left\{\begin{array}{l}
\frac{d^{2} u(t)}{d t^{2}}+A u(t)=f(t), \quad 0 \leq t \leq 1 \\
u(0)=\int_{0}^{1} \alpha(\rho) u(\rho) d \rho+\varphi \\
u_{t}(0)=\int_{0}^{1} \beta(\rho) u_{t}(\rho) d \rho+\psi
\end{array}\right.
$$

in a Hilbert space $H$ with a self-adjoint positive definite operator $A$. We are interested in studying the stability of solutions of problem (1) under the assumption

$$
\left|1+\int_{0}^{1} \alpha(s) \beta(s) d s\right|>\int_{0}^{1}(|\alpha(s)|+|\beta(s)|) d s .
$$

As in [30], a function $u(t)$ is called a solution of problem (1) if the following conditions are satisfied:

(i) $u(t)$ is twice continuously differentiable on the interval $(0,1)$ and continuously differentiable on the segment $[0,1]$.

(ii) The element $u(t)$ belongs to $D(A)$ for all $t \in[0,1]$, and the function $A u(t)$ is continuous on the segment $[0,1]$.

(iii) $u(t)$ satisfies the equation and nonlocal boundary conditions (1).

\section{The main theorem}

Let $H$ be a Hilbert space, $A$ be a positive definite self-adjoint operator with $A \geq \delta I$, where $\delta>\delta_{0}>0$. Throughout this paper, $\{c(t), t \geq 0\}$ is a strongly continuous cosine operatorfunction defined by

$$
c(t)=\frac{e^{i t A^{1 / 2}}+e^{-i t A^{1 / 2}}}{2} .
$$

Then, from the definition of sine operator-function $s(t)$,

$$
s(t) u=\int_{0}^{t} c(\rho) u d \rho
$$

it follows that

$$
s(t)=A^{-1 / 2} \frac{e^{i t A^{1 / 2}}-e^{-i t A^{1 / 2}}}{2 i} .
$$

For the theory of cosine operator-function we refer to [23] and [24].

Lemma 2.1 The following estimates hold:

$$
\|c(t)\|_{H \rightarrow H} \leq 1, \quad\left\|A^{1 / 2} s(t)\right\|_{H \rightarrow H} \leq 1 .
$$

Lemma 2.2 Suppose that assumption (2) holds. Then the operator T,

$$
T=\left(1+\int_{0}^{1} \int_{0}^{1} \alpha(s) \beta(s) d s d s\right) I-\int_{0}^{1}(\beta(s)+\alpha(s)) c(s) d s,
$$


has the inverse

$$
T^{-1}=\left[\left(1+\int_{0}^{1} \int_{0}^{1} \alpha(s) \beta(s) d s d s\right) I-\int_{0}^{1}(\beta(s)+\alpha(s)) c(s) d s\right]^{-1}
$$

and the following estimate is satisfied:

$$
\left\|T^{-1}\right\|_{H \rightarrow H} \leq \frac{1}{\left|1+\int_{0}^{1} \alpha(s) \beta(s) d s\right|-\int_{0}^{1}(|\alpha(s)|+|\beta(s)|) d s} .
$$

Proof Applying the triangle inequality and estimates (3), we obtain

$$
\begin{gathered}
\left\|\left(1+\int_{0}^{1} \int_{0}^{1} \alpha(s) \beta(s) d s d s\right) I-\int_{0}^{1}(\beta(s)+\alpha(s)) c(s) d s\right\|_{H \rightarrow H} \\
\geq\left|1+\int_{0}^{1} \alpha(s) \beta(s) d s\right|-\left\|\int_{0}^{1}(\beta(s)+\alpha(s)) c(s) d s\right\|_{H \rightarrow H} \\
\geq\left|1+\int_{0}^{1} \alpha(s) \beta(s) d s\right|-\int_{0}^{1}(|\alpha(s)|+|\beta(s)|)\|c(s)\|_{H \rightarrow H} d s \\
\geq\left|1+\int_{0}^{1} \alpha(s) \beta(s) d s\right|-\int_{0}^{1}(|\alpha(s)|+|\beta(s)|) d s>0 .
\end{gathered}
$$

Estimate (4) follows from this estimate. Lemma 2.2 is proved.

Now, we will obtain the formula for the solution of problem (1) for $\varphi \in D(A)$ and $\psi \in$ $D\left(A^{1 / 2}\right)$. It is clear that [23] the initial value problem

$$
\frac{d^{2} u}{d t^{2}}+A u(t)=f(t), \quad 0<t<1, \quad u(0)=u_{0}, \quad u^{\prime}(0)=u_{0}^{\prime}
$$

has a unique solution,

$$
u(t)=c(t) u_{0}+s(t) u_{0}^{\prime}+\int_{0}^{t} s(t-\lambda) f(\lambda) d \lambda
$$

where the function $f(t)$ is not only continuous but also continuously differentiable on $[0,1]$, $u_{0} \in D(A)$ and $u_{0}^{\prime} \in D\left(A^{1 / 2}\right)$.

Using (6) and the nonlocal boundary condition

$$
u_{0}=\int_{0}^{1} \alpha(y) u(y) d y+\varphi
$$

we get

$$
u_{0}=\int_{0}^{1} \alpha(y)\left(c(y) u_{0}+s(y) u_{0}^{\prime}+\int_{0}^{y} s(y-\lambda) f(\lambda) d \lambda\right) d y+\varphi
$$

Then

$$
\left(I-\int_{0}^{1} \alpha(y) c(y) d y\right) u_{0}-\int_{0}^{1} \alpha(y) s(y) d y u_{0}^{\prime}=\int_{0}^{1} \alpha(y) \int_{0}^{y} s(y-\lambda) f(\lambda) d \lambda d y+\varphi
$$


Differentiating both sides of (6), we obtain

$$
u^{\prime}(t)=-A s(t) u_{0}+c(t) u_{0}^{\prime}+\int_{0}^{t} c(t-\lambda) f(\lambda) d \lambda
$$

Using this formula and the integral condition

$$
u_{0}^{\prime}=\int_{0}^{1} \beta(y) u^{\prime}(y) d y+\psi
$$

we get

$$
u_{0}^{\prime}=\int_{0}^{1} \beta(y)\left(-A s(y) u_{0}+c(y) u_{0}^{\prime}+\int_{0}^{y} c(y-\lambda) f(\lambda) d \lambda\right) d y+\psi .
$$

Thus,

$$
\begin{gathered}
\int_{0}^{1} \beta(y) A s(y) d y u_{0}+\left(I-\int_{0}^{1} \beta(y) c(y) d y\right) u_{0}^{\prime} \\
=\int_{0}^{1} \beta(y) \int_{0}^{y} c(y-\lambda) f(\lambda) d \lambda d y+\psi
\end{gathered}
$$

Now, we have a system of equations (7) and (8) for the solution of $u_{0}$ and $u_{0}^{\prime}$. Solving it, we get

$$
\begin{aligned}
u_{0}= & T^{-1}\left\{\left[I-\int_{0}^{1} \beta(y) c(y) d y\right]\left[\int_{0}^{1} \alpha(y) \int_{0}^{y} s(y-\lambda) f(\lambda) d \lambda d y+\varphi\right]\right. \\
& \left.+\int_{0}^{1} \alpha(y) s(y) d y\left[\int_{0}^{1} \beta(y) \int_{0}^{y} c(y-\lambda) f(\lambda) d \lambda d y+\psi\right]\right\}
\end{aligned}
$$

and

$$
\begin{aligned}
u_{0}^{\prime}= & T^{-1}\left\{\left[I-\int_{0}^{1} \alpha(y) c(y) d y\right]\left[\int_{0}^{1} \beta(y) \int_{0}^{y} c(y-\lambda) f(\lambda) d \lambda d y+\psi\right]\right. \\
& \left.-\int_{0}^{1} \beta(y) A s(y) d y\left[\int_{0}^{1} \alpha(y) \int_{0}^{y} s(y-\lambda) f(\lambda) d \lambda d y+\varphi\right]\right\} .
\end{aligned}
$$

Hence, for the solution of the nonlocal boundary value problem (1) we have (6), (9), and (10).

Theorem 2.1 Suppose that $\varphi \in D(A), \psi \in D\left(A^{1 / 2}\right)$, and $f(t)$ is a continuously differentiable on $[0,1]$; assumption (2) holds. Then there is a unique solution of problem (1) and the following stability inequalities:

$$
\begin{aligned}
& \max _{0 \leq t \leq 1}\|u(t)\|_{H} \leq M\left[\|\varphi\|_{H}+\left\|A^{-1 / 2} \psi\right\|_{H}+\int_{0}^{1}\left\|A^{-1 / 2} f(\lambda)\right\|_{H} d \lambda\right], \\
& \max _{0 \leq t \leq 1}\left\|\frac{d u(t)}{d t}\right\|_{H}+\max _{0 \leq t \leq 1}\left\|A^{1 / 2} u(t)\right\|_{H} \\
& \quad \leq M\left[\left\|A^{1 / 2} \varphi\right\|_{H}+\|\psi\|_{H}+\int_{0}^{1}\|f(\lambda)\|_{H} d \lambda\right],
\end{aligned}
$$




$$
\begin{aligned}
& \max _{0 \leq t \leq 1}\left\|\frac{d^{2} u(t)}{d t^{2}}\right\|_{H}+\max _{0 \leq t \leq 1}\|A u(t)\|_{H} \\
& \quad \leq M\left\{\|A \varphi\|_{H}+\left\|A^{1 / 2} \psi\right\|_{H}+\|f(0)\|_{H}+\int_{0}^{1}\left\|f^{\prime}(\lambda)\right\|_{H} d \lambda\right\}
\end{aligned}
$$

are valid, where $M$ does not depend on $f(t), t \in[0,1], \varphi$, and $\psi$.

Proof We take the estimates

$$
\begin{aligned}
& \max _{0 \leq t \leq 1}\|u(t)\|_{H} \leq\left[\|u(0)\|_{H}+\left\|A^{-1 / 2} u^{\prime}(0)\right\|_{H}+\int_{0}^{1}\left\|A^{-1 / 2} f(t)\right\|_{H} d t\right], \\
& \max _{0 \leq t \leq 1}\left\|u^{\prime}(t)\right\|_{H}+\max _{0 \leq t \leq 1}\left\|A^{1 / 2} u(t)\right\|_{H} \\
& \quad \leq M\left[\left\|A^{1 / 2} u(0)\right\|_{H}+\left\|u^{\prime}(0)\right\|_{H}+\int_{0}^{1}\|f(t)\|_{H} d t\right], \\
& \max _{0 \leq t \leq 1}\left\|\frac{d^{2} u(t)}{d t^{2}}\right\|_{H}+\max _{0 \leq t \leq 1}\|A u(t)\|_{H}+\max _{0 \leq t \leq 1}\left\|A^{1 / 2} u^{\prime}(t)\right\|_{H} \\
& \quad \leq M\left[\|A u(0)\|_{H}+\left\|A^{1 / 2} u^{\prime}(0)\right\|_{H}+\|f(0)\|_{H}+\int_{0}^{1}\left\|f^{\prime}(t)\right\|_{H} d t\right]
\end{aligned}
$$

from [26] for the solution of problem (5). The proof of Theorem 2.1 is based on estimates (14), (15), (16), and the estimates for the norms of $u_{0}, A^{-1 / 2} u_{0}^{\prime}, A^{1 / 2} u_{0}, u_{0}^{\prime}, A u_{0}, A^{1 / 2} u_{0}^{\prime}$.

First of all, let us find an estimate for $\|u(0)\|_{H}$. By using (9) and estimates (3), (4), we obtain

$$
\|u(0)\|_{H} \leq M_{1}\left[\int_{0}^{1}\left\|A^{-1 / 2} f(\lambda)\right\|_{H} d \lambda+\|\varphi\|_{H}+\left\|A^{-1 / 2} \psi\right\|_{H}\right] .
$$

Applying $A^{-1 / 2}$ to (10), we get

$$
\begin{aligned}
A^{-1 / 2} u_{0}^{\prime}= & T^{-1}\left\{\left[I-\int_{0}^{1} \alpha(y) c(y) d y\right]\left[\int_{0}^{1} \beta(y) \int_{0}^{y} c(y-\lambda) A^{-1 / 2} f(\lambda) d \lambda d y+A^{-1 / 2} \psi\right]\right. \\
& -\int_{0}^{1} \beta(y) A^{1 / 2} s(y) d y \\
& \left.\times\left[\int_{0}^{1} \alpha(y) \int_{0}^{y} A^{1 / 2} s(y-\lambda) A^{-1 / 2} f(\lambda) d \lambda d y+\varphi\right]\right\} .
\end{aligned}
$$

Using estimates (3), (4), we obtain

$$
\left\|A^{-1 / 2} u^{\prime}(0)\right\|_{H} \leq M_{2}\left[\int_{0}^{1}\left\|A^{-1 / 2} f(\lambda)\right\|_{H} d \lambda+\left\|A^{-1 / 2} \psi\right\|_{H}+\|\varphi\|_{H}\right] .
$$

Thus, estimates (14), (17), and (18) yield estimate (11).

Second, applying operator $A^{1 / 2}$ to (9), we get

$$
\begin{aligned}
A^{1 / 2} u_{0}= & T^{-1}\left\{\left[I-\int_{0}^{1} \beta(y) c(y) d y\right]\left[\int_{0}^{1} \alpha(y) \int_{0}^{y} A^{1 / 2} s(y-\lambda) f(\lambda) d \lambda d y+A^{1 / 2} \varphi\right]\right. \\
& \left.+\int_{0}^{1} \alpha(y) A^{1 / 2} s(y) d y\left[\int_{0}^{1} \beta(y) \int_{0}^{y} c(y-\lambda) f(\lambda) d \lambda d y+\psi\right]\right\} .
\end{aligned}
$$


Using estimates (3) and (4), we obtain

$$
\left\|A^{1 / 2} u(0)\right\|_{H} \leq M_{1}\left[\int_{0}^{1}\|f(\lambda)\|_{H} d \lambda+\left\|A^{1 / 2} \varphi\right\|_{H}+\|\psi\|_{H}\right] .
$$

Using (10), and estimates (3), (4), we get

$$
\left\|u^{\prime}(0)\right\|_{H} \leq M_{2}\left[\int_{0}^{1}\|f(\lambda)\|_{H} d \lambda+\|\psi\|_{H}+\left\|A^{1 / 2} \varphi\right\|_{H}\right] .
$$

Then estimate (12) follows from estimates (15), (19), and (20).

Third, applying $A$ to (9) and using Abel's formula, we have

$$
\begin{aligned}
A u_{0}= & T^{-1}\left\{\left[I-\int_{0}^{1} \beta(y) c(y) d y\right]\right. \\
& \times\left[\int_{0}^{1} \alpha(y)\left[f(y)-c(y) f(0)-\int_{0}^{y} c(y-\lambda) f^{\prime}(\lambda) d \lambda\right] d y+A \varphi\right] \\
& +\int_{0}^{1} \alpha(y) A^{1 / 2} s(y) d y \\
& \left.\times\left[\int_{0}^{1} \beta(y)\left[A^{1 / 2} s(y) f(0)+\int_{0}^{y} A^{1 / 2} s(y-\lambda) f^{\prime}(\lambda) d \lambda\right] d y+A^{1 / 2} \psi\right]\right\}
\end{aligned}
$$

and using estimates (3), (4), we get

$$
\|A u(0)\|_{H} \leq M_{3}\left[\|A \varphi\|_{H}+\left\|A^{1 / 2} \psi\right\|_{H}+\|f(0)\|_{H}+\int_{0}^{1}\left\|f^{\prime}(\lambda)\right\|_{H} d \lambda\right] .
$$

In the same manner, applying $A^{1 / 2}$ to (10) and using Abel's formula, and estimates (3), (4), we obtain

$$
\left\|A^{1 / 2} u^{\prime}(0)\right\|_{H} \leq M_{4}\left[\|A \varphi\|_{H}+\left\|A^{1 / 2} \psi\right\|_{H}+\|f(0)\|_{H}+\int_{0}^{1}\left\|f^{\prime}(\lambda)\right\|_{H} d \lambda\right] .
$$

Thus, estimate (13) follows from estimates (16) and (21), and (22).

\section{Applications}

Now, we consider the applications of Theorem 2.1. First, a nonlocal boundary value problem for a hyperbolic equation

$$
\left\{\begin{array}{l}
u_{t t}-\left(a(x) u_{x}\right)_{x}+\sigma u=f(t, x), \quad 0<t<1,0<x<1, \\
u(0, x)=\int_{0}^{1} \alpha(\rho) u(\rho, x) d \rho+\varphi(x), \\
u_{t}(0, x)=\int_{0}^{1} \beta(\rho) u_{t}(\rho, x) d \rho+\psi(x), \quad 0 \leq x \leq 1, \\
u(t, 0)=u(t, 1), \quad u_{x}(t, 0)=u_{x}(t, 1), \quad 0 \leq t \leq 1
\end{array}\right.
$$

under assumption (2) is considered. Problem (23) has a unique smooth solution $u(t, x)$ for (2), smooth functions $a(x) \geq a>0(x \in(0,1)), a(0)=a(1), \varphi(x), \psi(x)(x \in[0,1])$ and $f(t, x)$ $(t, x \in[0,1]), \sigma$ a positive constant and under some conditions. This allows us to reduce problem (23) to nonlocal boundary value problem (1) in the Hilbert space $H=L_{2}[0,1]$ with a self-adjoint positive definite operator $A^{x}$ defined by (23). 
Theorem 3.1 For the solution of problem (23), we have the following stability inequalities:

$$
\begin{aligned}
& \max _{0 \leq t \leq 1}\left\|u_{x}(t, \cdot)\right\|_{L_{2}[0,1]} \leq M\left[\max _{0 \leq t \leq 1}\|f(t, \cdot)\|_{L_{2}[0,1]}+\left\|\varphi_{x}\right\|_{L_{2}[0,1]}+\|\psi\|_{L_{2}[0,1]}\right], \\
& \max _{0 \leq t \leq 1}\left\|u_{x x}(t, \cdot)\right\|_{L_{2}[0,1]}+\max _{0 \leq t \leq 1}\left\|u_{t t}(t, \cdot)\right\|_{L_{2}[0,1]} \\
& \quad \leq M\left[\max _{0 \leq t \leq 1}\left\|f_{t}(t, \cdot)\right\|_{L_{2}[0,1]}+\|f(0, \cdot)\|_{L_{2}[0,1]}+\left\|\varphi_{x x}\right\|_{L_{2}[0,1]}+\left\|\psi_{x}\right\|_{L_{2}[0,1]}\right],
\end{aligned}
$$

where $M$ does not depend on $\varphi(x), \psi(x)$, and $f(t, x)$.

The proof of Theorem 3.1 is based on Theorem 2.1 and the symmetry properties of the space operator generated by problem (23).

Proof Problem (23) can be written in the abstract form

$$
\left\{\begin{array}{l}
\frac{d^{2} u(t)}{d t^{2}}+A u(t)=f(t) \quad(0 \leq t \leq 1) \\
u(0)=\int_{0}^{1} \alpha(\rho) u(\rho) d \rho+\varphi \\
u_{t}(0)=\int_{0}^{1} \beta(\rho) u_{t}(\rho) d \rho+\psi
\end{array}\right.
$$

in the Hilbert space $L_{2}[0,1]$ of all square integrable functions defined on $[0,1]$ with a selfadjoint positive definite operator $A=A^{x}$ defined by the formula

$$
A^{x} u(x)=-\left(a(x) u_{x}\right)_{x}+\sigma u(x)
$$

with the domain

$$
D\left(A^{x}\right)=\left\{\begin{array}{c}
u(x): u, u_{x}\left(a(x) u_{x}\right)_{x} \in L_{2}[0,1] \\
u(0)=u(1), u^{\prime}(0)=u^{\prime}(1)
\end{array}\right\} .
$$

Here, $f(t)=f(t, x)$ and $u(t)=u(t, x)$ are known and unknown abstract functions defined on [0,1] with the values in $H=L_{2}[0,1]$. Therefore, estimates (24) and (25) follow from estimates (11), (12), and (13). Thus, Theorem 3.1 is proved.

Second, let $\Omega$ be the unit open cube in the $m$-dimensional Euclidean space $\mathbb{R}^{m}:\{x=$ $\left.\left(x_{1}, \ldots, x_{m}\right): 0<x_{j}<1,1 \leq j \leq m\right\}$ with boundary $S, \bar{\Omega}=\Omega \cup S$. In $[0,1] \times \Omega$, let us consider a boundary value problem for the multidimensional hyperbolic equation

$$
\left\{\begin{array}{l}
\frac{\partial^{2} u(t, x)}{\partial t^{2}}-\sum_{r=1}^{m}\left(a_{r}(x) u_{x_{r}}\right)_{x_{r}}+\sigma u(x)=f(t, x), \\
\quad x=\left(x_{1}, \ldots, x_{m}\right) \in \Omega, 0<t<1, \\
u(0, x)=\int_{0}^{1} \alpha(\rho) u(\rho, x) d \rho+\varphi(x), \quad x \in \bar{\Omega}, \\
u_{t}(0, x)=\int_{0}^{1} \beta(\rho) u_{t}(\rho, x) d \rho+\psi(x), \quad x \in \bar{\Omega}, \\
u(t, x)=0, \quad x \in S,
\end{array}\right.
$$

under assumption (2). Here, $a_{r}(x)(x \in \Omega), \varphi(x), \psi(x)(x \in \bar{\Omega})$ and $f(t, x), t \in(0,1), x \in \Omega$ are given smooth functions and $a_{r}(x) \geq a>0$. 
Let us introduce the Hilbert space $L_{2}(\bar{\Omega})$ of all square integrable functions defined on $\bar{\Omega}$, equipped with the norm

$$
\|f\|_{L_{2}(\bar{\Omega})}=\left\{\int \cdots \int_{x \in \bar{\Omega}}|f(x)|^{2} d x_{1} \cdots d x_{m}\right\}^{\frac{1}{2}} .
$$

Theorem 3.2 For the solution of problem (27), the following stability inequalities hold:

$$
\begin{aligned}
& \max _{0 \leq t \leq 1} \sum_{r=1}^{m}\left\|u_{x_{r}}(t, \cdot)\right\|_{L_{2}(\bar{\Omega})} \\
& \leq M\left[\max _{0 \leq t \leq 1}\|f(t, \cdot)\|_{L_{2}(\bar{\Omega})}+\sum_{r=1}^{m}\left\|\varphi_{x_{r}}\right\|_{L_{2}(\bar{\Omega})}+\|\psi\|_{L_{2}(\bar{\Omega})}\right] \\
& \max _{0 \leq t \leq 1} \sum_{r=1}^{m}\left\|u_{x_{r} x_{r}}(t, \cdot)\right\|_{L_{2}(\bar{\Omega})}+\max _{0 \leq t \leq 1}\left\|u_{t t}(t, \cdot)\right\|_{L_{2}(\bar{\Omega})} \\
& \leq M\left[\max _{0 \leq t \leq 1}\left\|f_{t}(t, \cdot)\right\|_{L_{2}(\bar{\Omega})}+\|f(0, \cdot)\|_{L_{2}(\bar{\Omega})}\right. \\
& \left.\quad+\sum_{r=1}^{m}\left\|\varphi_{x_{r} x_{r}}\right\|_{L_{2}(\bar{\Omega})}+\sum_{r=1}^{m}\left\|\psi_{x_{r}}\right\|_{L_{2}(\bar{\Omega})}\right]
\end{aligned}
$$

where $M$ does not depend on $\varphi(x), \psi(x)$, and $f(t, x)(t \in(0,1), x \in \Omega)$.

Proof Problem (27) can be written in the abstract form (26) in the Hilbert space $L_{2}(\bar{\Omega})$ with a self-adjoint positive definite operator $A=A^{x}$ defined by the formula

$$
A^{x} u(x)=-\sum_{r=1}^{m}\left(a_{r}(x) u_{x_{r}}\right)_{x_{r}}+\sigma u(x)
$$

with domain

$$
D\left(A^{x}\right)=\left\{u(x): u, u_{x_{r}},\left(a_{r}(x) u_{x_{r}}\right)_{x_{r}} \in L_{2}(\bar{\Omega}), 1 \leq r \leq m, u(x)=0, x \in S\right\} .
$$

Here, $f(t)=f(t, x)$ and $u(t)=u(t, x)$ are known and unknown abstract functions defined on $\bar{\Omega}$ with the values in $H=L_{2}(\bar{\Omega})$. So, estimates (28) and (29) follow from estimates (11), (12), (13), and the following theorem.

Theorem 3.3 [29] For the solution of the elliptic differential problem

$$
\begin{aligned}
& A^{x} u(x)=\omega(x), \quad x \in \Omega, \\
& u(x)=0, \quad x \in S,
\end{aligned}
$$

the following coercivity inequality holds:

$$
\sum_{r=1}^{m}\left\|u_{x_{r} x_{r}}\right\|_{L_{2}(\bar{\Omega})} \leq M\|\omega\|_{L_{2}(\bar{\Omega})}
$$

where $M$ is independent of $\omega$. 


\section{Conclusion}

This work is devoted to the study of the stability of the nonlocal boundary value problem with integral conditions for hyperbolic equations. For the solution of nonlocal boundary problem (1) in a Hilbert space $H$ with a self-adjoint positive definite operator $A$, Theorem 2.1 is established. Two applications of Theorem 2.1 are given. Of course, stable twostep difference schemes for approximate solution of problem (1) can be presented. The methods given above permit us to establish the stability of these difference schemes. Applying [31], we can give a numerical support of the theoretical results.

Competing interests

The authors declare that they have no competing interests.

\section{Authors' contributions}

All authors contributed equally to the writing of this paper. All authors read and approved the final manuscript.

\section{Author details}

'Department of Mathematics, Fatih University, Istanbul, 34500, Turkey. ${ }^{2}$ Department of Mathematics, ITTU, Gerogly Street, Ashgabat, Turkmenistan.

\section{Acknowledgements}

This work is supported by the Scientific Research Fund of Fatih University (Project No: P50041203-B).

Received: 15 June 2014 Accepted: 19 August 2014 Published online: 25 September 2014

\section{References}

1. Shi, P: Weak solution to evolution problem with a nonlocal constraint. SIAM J. Math. Anal. 24, 46-58 (1993)

2. Choi, YS, Chan, KY: A parabolic equation with nonlocal boundary conditions arising from electrochemistry. Nonlinear Anal. 18, 317-331 (1992)

3. Cahlon, B, Kulkarni, DM, Shi, P: Stepwise stability for the heat equation with a nonlocal constraint. SIAM J. Numer. Anal. 32, 571-593 (1995)

4. Samarski, AA: Some problems in the modern theory of differential equations. Differ. Uravn. 16, 1221-1228 (1980)

5. Beilin, SA: Existence of solutions for one-dimensional wave equations with non-local conditions. Electron. J. Differ. Equ. 2001, 76 (2001)

6. Dehghan, M: On the solution of an initial-boundary value problem that combines Neumann and integral condition for the wave equation. Numer. Methods Partial Differ. Equ. 21, 24-40 (2005)

7. Avalishvili, G, Avalishvili, M, Gordeziani, D: On integral nonlocal boundary value problems for some partial differential equations. Bull. Georgian Acad. Sci. 5(1), 31-37 (2011)

8. Pulkina, LS: A nonlocal problem with integral conditions for hyperbolic equations. Electron. J. Differ. Equ. 1999, 45 (1999)

9. Ramezani, M, Dehghan, M, Razzaghi, M: Combined finite difference and spectral methods for the numerical solution of hyperbolic equation with an integral condition. Numer. Methods Partial Differ. Equ. 24, 1-8 (2008)

10. Pulkina, LS: On solvability in $L_{2}$ of nonlocal problem with integral conditions for hyperbolic equations. Differ. Uravn. 36(2), 279-280 (2000)

11. Mesloub, S, Bouziani, A: On a class of singular hyperbolic equation with a weighted integral condition. Int. J. Math. Math. Sci. 22(3), 511-519 (1999)

12. Shakeri, F, Dehghan, M: The method of lines for solution of the one-dimensional wave equation subject to an integral conservation condition. Comput. Math. Appl. 56, 2175-2188 (2008)

13. Dehghan, $M$, Saadatmandi, $A$ : Variational iteration method for solving the wave equation subject to an integral conservation condition. Chaos Solitons Fractals 41, 1448-1453 (2009)

14. Dehghan, M, Shokri, A: A meshless method for numerical solution of the one-dimensional wave equation with an integral condition using radial basis functions. Numer. Algorithms 52, 461-477 (2009)

15. Dehghan, M, Saadatmandi, A: Numerical solution of the one-dimensional wave equation with an integral condition. Numer. Methods Partial Differ. Equ. 23, 282-292 (2007)

16. Yousefi, SA, Barikbin, Z, Dehghan, M: Bernstein Ritz-Galerkin method for solving an initial-boundary value problem that combines Neumann and integral condition for the wave equation. Numer. Methods Partial Differ. Equ. 26, 1236-1246 (2010)

17. Ashyralyev, A, Aggez, N: A note on the difference schemes of the nonlocal boundary value problems for hyperbolic equations. Numer. Funct. Anal. Optim. 25(5-6), 1-24 (2004)

18. Sobolevskii, PE, Semenov, S: On some approach to investigation of singular hyperbolic equations. Dokl. Akad. Nauk SSSR 270(1), 555-558 (1983)

19. Agarwal, RP, Bohner, M, Shakhmurov, VB: Maximal regular boundary value problems in Banach-valued weighted space. Bound. Value Probl. 1, 9-42 (2004)

20. Ashyralyev, A, Sobolevskii, PE: New Difference Schemes for Partial Differential Equations. Birkhäuser, Basel (2004)

21. Ashyralyev, A, Sobolevskii, PE: Well-Posedness of Parabolic Difference Equations. Operator Theory Advances and Applications. Birkhäuser, Basel (1994)

22. Kostin, VA: On analytic semigroups and strongly continuous cosine functions. Dokl. Akad. Nauk SSSR 307(4), 796-799 (1989) 
23. Fattorini, HO: Second Order Linear Differential Equations in Banach Space. Notas de Matematica. North-Holland, Amsterdam (1985)

24. Piskarev, S, Shaw, Y: On certain operator families related to cosine operator function. Taiwan. J. Math. 1(4), 3585-3592 (1997)

25. Guidetti, D, Karasözen, B, Piskarev, S: Approximation of abstract differential equations. J. Math. Sci. 122(2), $3013-3054$ (2004)

26. Ashyralyev, A, Sobolevskii, PE: A note on the difference schemes for hyperbolic equations. Abstr. Appl. Anal. 6(2), 63-70 (2001)

27. Soltanov, H: A note on the Goursat problem for a multidimensional hyperbolic equation. Contemp. Anal. Appl. Math. 1(2), 98-106 (2013)

28. Ashyralyev, A, Aggez, N: Finite difference method for hyperbolic equations with the nonlocal integral condition. Discrete Dyn. Nat. Soc. 2011, 562385 (2011)

29. Sobolevskii, PE: Difference Methods for the Approximate Solution of Differential Equations. Izdat. Voronezh. Gosud. Univ., Voronezh (1975)

30. Ashyralyev, A, Yildirim, O: On multipoint nonlocal boundary value problems for hyperbolic differential and difference equations. Taiwan. J. Math. 14(1), 165-194 (2010)

31. Ashyralyev, A, Aggez, N: On the solution of NBVP for multidimensional hyperbolic equations. Sci. World J. 2014 841602 (2014). doi:10.1155/2014/841602

doi:10.1186/s13661-014-0205-4

Cite this article as: Ashyralyev and Aggez: Nonlocal boundary value hyperbolic problems involving integral conditions. Boundary Value Problems 2014 2014:205.

\section{Submit your manuscript to a SpringerOpen ${ }^{\circ}$ journal and benefit from:}

- Convenient online submission

- Rigorous peer review

- Immediate publication on acceptance

- Open access: articles freely available online

- High visibility within the field

- Retaining the copyright to your article 